\title{
UNA PROPUESTA PEDAGÓGICA PARA EL DESARROLLO DE LA COMPETENCIA PRAGMÁTICA EN ESPAÑOL COMO SEGUNDA LENGUA O LENGUA EXTRANJERA
}

\author{
María Soledad Urbina Vargas ${ }^{1}$
}

Recibido:7/08/2008

Aceptado: 24/10/2008

\section{Resumen}

Este artículo analiza el papel de la enseñanza en el desarrollo de la competencia pragmática en aprendices de español como segunda lengua o lengua extranjera. Se propone el modelo pedagógico de enseñanza metapragmática explícita, el cual es implementado mediante actividades de input: video grabaciones y muestras escritas de lenguaje auténtico en la lengua meta, y actividades de output: video grabaciones de simulaciones hechas por los estudiantes, de los actos de habla seleccionados para el proceso de enseñanza aprendizaje en la clase de segunda lengua.

\section{Palabras clave \\ -Pragmática •Competencia comunicativa •Actos de habla •Lengua meta •Enseñanza explícita}

\begin{abstract}
This article analyses the role of education in the pragmatic competition development in Spanish as a second language or foreign language. It proposes the explicit metapragmatic instruction pedagogical model, which is implemented through input activities: video recordings and written samples of authentic language in the target language, and output activities: video recordings of simulations made by students, of speech acts selected for the teaching-learning process in the class of second language.
\end{abstract}

\section{Key words}

$\bullet$ Pragmatic $\bullet$ Communicative competence $\bullet$ Speech Acts $\bullet$ Target language $\bullet$ Explicit teaching

\footnotetext{
1 Editora Revista Biocenosis. Centro de Educación Ambiental, Universidad Estatal a Distancia. Investigadora y educadora en el área del español como segunda lengua. surbina@uned.ac.cr/ soledad.urbina@gmail.com
} 


\section{INTRODUCCIÓN}

A principios de los años 80, la Enseñanza del Español como Segunda Lengua (ESL) comienza a consolidarse en Costa Rica, tanto a nivel de mayor afluencia de estudiantes extranjeros, como de creación de instituciones educativas privadas dedicadas a la docencia en esta área. Poco tiempo después, son las instituciones públicas como la Universidad de Costa Rica o la Universidad Nacional las que abren sus puertas al desarrollo de esta disciplina, primero, ofreciendo cursos de lengua para estudiantes extranjeros; más tarde, formando profesionales capacitados para la docencia y la investigación del español como segunda lengua y como lengua extranjera ${ }^{1}$.

Por ser la lingüística aplicada a la enseñanza del español una área de desarrollo relativamente nuevo en nuestro país, es necesario encausar su avance hacia el empleo de los conocimientos actuales en el campo de la adquisición de segundas lenguas (ASL) y lenguas extranjeras, los cuales abarcan disciplinas tales como la lingüística, la sociología, la lexicografía, la pragmática, la antropología, entre otras materias.

Este trabajo plantea la aplicación de una propuesta didáctica para que los aprendices de español como segunda lengua o lengua extranjera desarrollen su competencia pragmática en cuanto a interpretación de enunciados y su producción, en situaciones y contextos específicos.

Pero, ¿qué contempla la Pragmática?, Levinson (1983) propone que es el estudio de aquellas relaciones entre lengua y contexto, gramaticalizadas o codificadas en la estructura de la lengua. Si tomamos esta definición como un punto de partida para entender el objeto de la Pragmática, podemos extrapolar que en este campo de estudio se 
caracterizan los enunciados valorando su adecuación dentro del contexto y la situación en que se emiten.

Dentro de la Pragmática se han priorizado los estudios sobre la cortesía verbal, con el propósito de explicar las formas lingüísticas que se emplean para realizar los diversos actos de habla: unidades básicas de la comunicación, mediante las cuales se realiza una acción o acto social específico (p. ej. invitar, afirmar, rechazar, pedir, aconsejar) ${ }^{2}$, y los factores del contexto que determinan su selección.

Las intenciones de los hablantes, la interpretación del enunciado y la relación entre los participantes (mayor o menor distancia social, afecto, entre otros) son elementos propios de la cortesía verbal. También le es atinente el porqué a veces se expresa algo queriendo decir otra cosa, con quién y en qué situación es adecuada la utilización de un enunciado. La necesidad de incluir el desarrollo de la competencia pragmática dentro del currículo del español como segunda lengua (EL2) o lengua extranjera es un planteamiento que ha motivado investigaciones tanto en el campo teórico del proceso de adquisición (el cómo se desarrolla la habilidad pragmática en una segunda lengua), como en el campo de la enseñanza-aprendizaje (el entender, facilitar y usar los elementos pragmáticos de la lengua por adquirir); no obstante, para Kasper y Rose (2002), la mayoría de los estudios están orientados más hacia el uso de las habilidades pragmáticas y no hacia el proceso de adquisición de estas.

Con respecto a la enseñanza- aprendizaje de segundas lenguas o lenguas extranjeras, las investigaciones en el campo de los actos de habla (Austin, 1962; Searle, 1975), las implicaturas conversacionales (Grice, 1975), el principio de cortesía de Leech (1983) y el modelo de cortesía de Brown y Levinson (1987), brindaron la apertura hacia la 
observación de la competencia pragmática como uno de los aspectos que conforma la competencia comunicativa, ya que impulsan el desarrollo de estudios de las semejanzas y diferencias en la realización de actos de habla y de la cortesía en distintas lenguas y culturas. Cobra entonces importancia el mostrar a los aprendices de segunda lengua, inmersos en una cultura específica, las circunstancias en las cuales un enunciado puede o no seleccionarse dentro del contexto sociocultural en el que se desenvuelve y cuál es su valor dentro del mismo contexto.

Si el hablante no nativo $(\mathrm{HnN})$, al comunicarse, no tiene la capacidad de utilizar y comprender adecuadamente la lengua en contexto, tomando en cuenta la relación entre los participantes, además de otros factores como la intención, la situación comunicativa y el aspecto cultural, posiblemente se verá muy limitado al hacer la selección de los enunciados apropiados que le permitan una comunicación efectiva, es decir, el emplear con mayor propiedad la lengua meta, lo más próximo a como lo haría un hablante nativo.

\section{Algunos estudios pragmáticos sobre cortesía verbal en español como segunda lengua}

Dentro del área curricular del español como segunda lengua o como lengua extranjera, Guillermina Piatti (2003) propone la inclusión de la cortesía en los contenidos de los programas de lengua. En el estudio de Piatti se contrastan las estrategias de cortesía empleadas por hablantes nativos y no nativos del español argentino (como grupo de referencia) al dar opiniones, hacer pedidos, reclamos o disculparse. Conforme con los resultados, la autora señala, en el análisis de la muestra de $\mathrm{HnN}$, la utilización generalizada de algunas expresiones por desconocimiento de otras, la inseguridad al intervenir y la falta de adecuación al contexto y propone una serie de actividades encaminadas a solventar este faltante. 
En la misma área de investigación, Domnita Dumitrescu (2005) realizó un estudio comparativo entre estudiantes californianos nativos, no nativos y bilingües (por herencia) del español, sobre el acto de habla cortés de agradecer y la respuesta al agradecimiento que, al igual que el de Piatt, señala el pobre manejo de las estrategias, además de las constantes transferencia del inglés al español por parte de los $\mathrm{HnN}$ y bilingües.

Por su parte, Murillo (2004) realiza un recuento de las principales nociones de la pragmática, para luego relacionarlas con los enfoques de enseñanza-aprendizaje de segundas lenguas.

En su estudio, Murillo analiza la competencia pragmática de estudiantes de nivel avanzado al hacer peticiones o dar consejos. Observa que el uso de estrategias de atenuación es muy variado y en algunos casos no corresponde el nivel de proficiencia (empleo de estructuras gramaticales o léxicas) con el nivel de competencia pragmática. Su inquietud se encamina a la falta de valoración del componente pragmático en el currículo de los cursos de EL2, su enseñanza- aprendizaje y su evaluación.

Según el investigador, se debe fomentar la elaboración de propuestas curriculares (en español como segunda lengua) que involucren el componente pragmático, basadas en una teoría de la adquisición pragmática, en una reflexión sobre la enseñabilidad o no de todos o algunos aspectos pragmáticos y en cuáles enfoques metodológicos son los más aptos para introducir a los aprendices en esta dimensión comunicativa.

Los resultados que presentan estos investigadores precitados señalan la falta de adecuación pragmática, por parte de hablantes no nativos del español, en situaciones comunicativas específicas. 


\section{La cortesía verbal en libros para la enseñanza del español como segunda lengua o lengua extranjera:}

Sara Bani y Almudena Nevado (2004) analizan el tratamiento de la cortesía verbal en ocho manuales de enseñanza de español para extranjeros y sus respectivos manuales de ejercicios y en seis gramáticas, también comúnmente empleadas en el proceso de enseñanza-aprendizaje del español.

Plantean que, en las gramáticas, todas de enfoque comunicativo ${ }^{3}$, se pueden encontrar elementos gramaticales que son asociados con el concepto de cortesía, tales como el uso de los pronombres y el empleo de formas verbales asociadas con el uso cortés (condicional e imperativo). Las autoras parten de estos elementos gramaticales para también observar su tratamiento desde la perspectiva pragmática de la cortesía.

Bani y Nevado encuentran una serie de limitaciones en las obras analizadas con respecto al tratamiento de la cortesía de los temas en estudio: si bien estas obras los contienen como elementos de cortesía, todos carecen de explicaciones amplias, presentan falta de contextualización, se da prioridad al elemento lingüístico sobre el pragmático o se explican brevemente y sin ejercicios que ayuden a fijar el conocimiento.

Las investigadoras proponen un tratamiento de la cortesía que permee toda la actividad comunicativa y, a la vez, brindan una serie de ejercicios en los que el estudiante, mientras revisa las formas lingüísticas, reflexiona sobre los elementos pragmáticos que envuelve la interacción entre los participantes en un intercambio comunicativo.

Dentro de la misma línea de investigación, Horacio Miranda Ubilla (2000) realiza un estudio sobre la cortesía verbal en textos para la enseñanza del español y del inglés como lenguas extranjeras. Presenta los aspectos teóricos más relevantes que sustentan el concepto de cortesía, hace un recuento de los métodos de enseñanza de lenguas y analiza 
la metodología empleada y el tratamiento de la cortesía en seis manuales de enseñanza de lenguas.

Los textos fueron seleccionados por el enfoque comunicativo empleado en cada uno, método que para Miranda Ubilla privilegia el uso de estructuras relacionadas con la cortesía verbal.

Miranda analiza la presencia de la cortesía verbal en los textos didácticos completos. Su estudio abarca todas las actividades pedagógicas presentes en los libros (instrucciones, tareas, ejercicios, títulos de unidades) en las que de algún modo podría estar incorporado el tema.

Para el investigador, los actos corteses sí tienen presencia en la selección pero se presentan de una forma en la que el propósito no es el tema de la cortesía, sino la enseñanza de estructuras gramaticales, funciones comunicativas o aspectos culturales, es decir, no aparece como un contenido programático propio en ningún texto.

Los autores concuerdan en que aun cuando las obras contienen elementos de cortesía, carecen de explicaciones amplias, y se nota una falta de contextualización de los ejercicios de prácticas, además de la prioridad del elemento lingüístico sobre el pragmático.

\section{Enseñanza - aprendizaje de la competencia pragmática}

Al dejar atrás los métodos estructuralistas de enseñanza del español como segunda lengua o lengua extranjera, como principal opción didáctica (aquellos en que se priorizaba el estudio gramatical de la lengua restando protagonismo a la lengua en uso), los profesores de segunda lengua han implementado en sus programas elementos del enfoque comunicativo, con la ganancia de priorizar además de la adquisición de las formas 
lingüísticas, la transmisión del contenido, las relaciones entre los enunciados, los hablantes, las intenciones de éstos y las situaciones o contextos culturales en que se desarrolla la interacción. En otras palabras, se busca que el aprendiz adquiera la capacidad de resolver situaciones y necesidades comunicativas.

Dentro de esta misma concepción, la enseñanza de la pragmática en segundas lenguas está dirigida a desarrollar la habilidad del aprendiz en el empleo de un lenguaje social apropiado según la situación comunicativa en la que se involucre. Los contenidos específicamente pragmáticos deben presentarse de forma integrada con el resto de los elementos propios de la lengua: gramática, fonética, semántica, etc.

Kasper (1997) considera que los aprendices adultos de una segunda lengua poseen mucha información pragmática, sin embargo no siempre la utilizan. Señala que el papel de la instrucción no es tanto el brindar nueva información, sino el lograr que este conocimiento pragmático previo de su primera, o de otras lenguas que domine, sea transferido a los contextos comunicativos en la lengua meta, es decir, que pueda emplear los rasgos generales de la pragmática de su primera lengua adaptándolos a las nuevas estructuras lingüísticas de la lengua por adquirir, mediante ejercicios que concienticen a los estudiantes de la conveniencia de aplicar sus conocimientos pragmáticos universales.

En el campo de los estudios de adquisición de segundas lenguas o lenguas extranjeras, el desarrollo de la competencia pragmática ha promovido investigaciones en temas como qué tipo de instrucción deben recibir los aprendices, instrucción institucional versus aprendizaje por exposición al medio, o en cuál nivel de interlengua ${ }^{4}$ la competencia pragmática debe ser parte del currículo. 
Varios estudios que recogen en su compilación Kasper y Rose dan cuenta de que la gran mayoría de las características pragmáticas de las segundas lenguas son enseñables: “(...) estas incluyen una variedad de aspectos discursivos, pragmáticos y sociolingüísticos de la enseñanza, tales como marcas discursivas y estrategias, rutinas pragmáticas, actos de habla, características discursivas y comprensión pragmática (...)”. (Nuestra traducción), (Kasper y Rose, 2002, p. 269).

También se plantean las posibilidades que tiene un aprendiz de segunda lengua en cuanto al acceso a la información pragmática de la lengua meta. El hablante no nativo podrá adquirir los conocimientos pragmáticos a través de dos vías: del medio en el cual se desarrolla y mediante la instrucción formal. Con respecto a las posibilidades “(...) parece ser (con base en las investigaciones hechas al respecto), que los hablantes que reciben instrucción tienen más éxito que aquellos que no" (Kasper y Rose, 2002, p.269).

(...) la mayoría de los resultados de los estudios del efecto de la instrucción están en completo acuerdo con las investigaciones que muestran que, sin instrucción formal, los aprendices no logran suficiente habilidad en el área pragmática. (Nuestra traducción), (Bardovi-Harlig, 2001, citada en Kasper y Rose, 2002, p.238).

En relación con los diferentes enfoques al enseñar la pragmática, encontramos dos vías: una es la enseñanza explícita, es decir, cuando las explicaciones de las reglas forman parte de la instrucción y a los aprendices se les pide analizar una forma particular y llegar a generalizaciones metapragmáticas (enfatizando en factores que inciden en la escogencia de una forma específica: relaciones sociales, aspectos culturales) y, por el contrario, cuando ni la explicación de las reglas ni la reflexión metapragmática de una forma son 
parte del proceso, la enseñanza es considerada implícita ( en cierto sentido una orientación de exposición hacia las estructuras y no hacia el contexto de uso).

La mayoría de los estudios recogidos en Kasper y Rose (2002) sobre este aspecto demuestran que la instrucción explícita de las normas, junto con un input adecuado y una amplia práctica de los conocimientos adquiridos, brindan los mejores resultados en el proceso de aprendizaje de esta competencia.

(...) La instrucción metapragmática generalmente es combinada con la discusión metapragmática, lo cual requiere una participación activa del estudiante en el formato de presentación por parte del profesor o en grupos. Muchas de las investigaciones se han orientado hacia la eficacia de la instrucción explícita comparándola con otras estrategias de instrucción, en especial a nivel de enseñanza explícita y enseñanza implícita. Varios trabajos (...) han comparado la instrucción metapragmática con las condiciones únicas de input y práctica. La mayoría de estos estudios encuentran ventajas al emplear la instrucción metapragmática. (Nuestra traducción), (p.53).

Urbina (2007) analiza la enunciación de los actos de aceptar o rechazar la invitación por parte de dos muestras de hablantes no nativos del español, con el propósito de valorar los efectos que produce la instrucción metapragmática explícita aplicada a una de las muestras.

En una primera fase, se recogió el discurso de un grupo de 18 hablantes nativos del español vallecentraleño ${ }^{5}$, con el propósito de emplear los enunciados elicitados como parámetro de respuesta al aceptar o rechazar una invitación y usar esta información para 
la elaboración del material didáctico utilizado en la enseñanza explícita de los actos de habla en estudio.

La población de hablantes no nativos del español incluyó dos muestras, cada una integrada por 18 participantes, aprendices de español como segunda lengua con un nivel principiante de competencia y expuestos a un mismo programa curricular, con la salvedad de que la primera muestra fue expuesta, durante dos horas y media (2:30), a una instrucción metapragmática explícita de los actos de habla en estudio; mientras que la segunda (grupo control) recibió instrucción sobre los actos de habla de tipo léxico y gramatical, atendiendo solo a su formulación en ejercicios de lectura y práctica de diálogos, sin ninguna instrucción metapragmática explícita y con un menor tiempo de exposición (50 minutos).

El análisis de los datos de ambas muestras reveló que los enunciados de los hablantes no nativos que recibieron instrucción explícita de los actos de habla en estudio, presentaban una mayor adecuación pragmática con respecto a las de los hablantes no nativos que no fueron expuestos a ese tipo de enseñaza; adecuación en cuanto a que los patrones de respuesta y la variedad empleada de actos de habla y subactos de habla ${ }^{6}$, coincidieron en mayor grado con los obtenidos con base en la muestra de los hablantes nativos del español o grupo de referencia.

Por otra parte, la aplicación del instrumento en un grupo de estudiantes de español como segunda lengua fue una ventaja, ya que esa circunstancia promovió el que los aprendices fueran más observadores de las categorías y las convenciones sociales establecidas para manifestar diferentes tipos de actos de habla dentro de la cultura meta. Además, ambos grupos de no nativos estuvieron expuestos al medio cultural costarricense, a un mismo 
input natural, lo cual hizo más evidente el papel de la instrucción como marcadora de diferencias para desarrollar habilidades en esta competencia.

Otra interrogante fundamental, en el área de la adquisición de la pragmática, es en qué nivel de interlengua o estadio de adquisición del estudiante debe ser desarrollada la competencia pragmática en los procesos de instrucción en segundas lenguas. Para Bardovi-Harlig y Mahan-Taylor (2003), la pragmática puede ser integrada en los currículos de segunda lengua desde niveles iniciales, y así reducir el desequilibrio entre el desarrollo gramatical y el pragmático en la instrucción.

Wildner- Bassett (1994, citado por Tateyama, 2001) y Tateyama $(1997 ; 2001)$, en sus investigaciones sobre el aprendizaje del alemán y el japonés, respectivamente, demostraron que el empleo de rutinas pragmáticas en contexto puede desarrollarse en estudiantes de niveles básicos de competencia, lo cual para Kasper (1997) representa un hallazgo importante en el diseño de currículos y programas ya que deja atrás el mito de que la competencia pragmática sólo puede ser integrada cuando se cuenta con sólidas bases gramaticales y, por el contrario, la necesidad de entender y expresar los mensajes impulsa el aprendizaje de nuevas formas lingüísticas.

\section{Instrumento pedagógico propuesto}

Al partir de la premisa de Kasper y Rose (2002), basada en varios trabajos de investigación en el área de la adquisición de la competencia pragmática, de que diferentes tipos de instrucción proveen diferentes resultados, se propone la metodología de enseñanza explícita.

Para presentar a los aprendices el concepto de cortesía y las estrategias empleadas en los actos de habla por mostrar, se deben tener en cuenta varios factores: 
a) El nivel de competencia lingüística ${ }^{7}$ de los aprendices.

b) La relación entre las explicaciones explícitas (metapragmáticas) y los ejercicios de práctica.

c) El equilibrio entre el aspecto formal de un elemento lingüístico y su uso; generalmente se privilegia la funcionalidad del elemento dentro del contexto comunicativo.

En términos del instrumento pedagógico planteado, la instrucción metapragmática explícita se refiere a la reflexión, descripción, explicación y discusión de las características pragmáticas meta, mediante actividades de input (orales y escritas) y con la puesta en práctica de actividades orientadas a fijar la información recibida mediante la práctica oral y escrita (actividades de output), es decir, actividades destinadas a despertar la conciencia pragmática del estudiante y actividades de práctica comunicativa.

\section{Actividades y técnicas comunicativas sugeridas para la enseñanza metapragmática explícita de la cortesía verbal}

La escogencia de los actos de habla por presentar en una clase de segunda lengua no es al azar, sino por la posibilidad de ser enseñados según el nivel de competencia comunicativa de los aprendices. En el entendido de que la competencia pragmática, como cualquier otra, se va desarrollando progresivamente ${ }^{8}$.

Se recomienda emplear varias actividades, de naturaleza comunicativa en su mayoría, para desarrollar y fijar las estructuras meta para expresar la cortesía en español. A continuación se describen algunas actividades y técnicas sugeridas:

1) Uso de muestras de lenguaje auténtico, actividad de input: observación de videos cortos, de interacciones reales. Mediante esta práctica de observación de videos 
cortos tanto de las situaciones por analizar como de otras similares (por ejemplo, distintas relaciones entre los participantes, mayor o menor grado de afecto, jerarquía, etc.), los estudiantes pueden fijar su atención en las estructuras en estudio y reconocerlas en situaciones similares.

2) Lectura y dramatización de diálogos: otra vía de presentación del input de forma escrita y a la vez una posibilidad de práctica oral de los enunciados para manifestar los actos de habla en estudio, mediante diversas situaciones comunicativas.

3) Video-grabación de interacciones de los estudiantes (actividad de output): mediante la simulación de situaciones relacionadas con el acto de habla en estudio se persigue una retroalimentación, al ser estas analizadas luego por los estudiantes y el profesor.

La manipulación del input ofrece variedad de recursos didácticos: la detección de las variables sociopragmáticas como la distancia social entre los participantes, los gestos, las variables situacionales, la entonación de los enunciados, el tipo de estructura lingüística empleada en cada interacción y el empleo del recurso para reelaborar situaciones parecidas.

Los estudiantes reflexionan sobre las diferencias y semejanzas entre la cultura del aprendiz y la cultura meta, sobre las conductas apropiadas al expresar los enunciados y en cómo una conducta no aceptada podría prestarse a malentendidos, a valoraciones negativas por parte de los hablantes nativos de la lengua meta y a crear estereotipos culturales que provoquen juicios como "los estadounidenses son menos educados" o "los europeos son menos expresivos", es decir, como mecanismo efectivo para incrementar su conciencia pragmática. 
Acorde con Bardovi-Harlig y Mahan-Taylor (2003), la presentación de lenguaje auténtico debe preceder las actividades de interpretación y producción con el fin de proveer a los aprendices de una base de conocimientos.

Una recomendación para poner en práctica las actividades propuestas es trabajar con grupos pequeños (entre 6 y 8 estudiantes) y en cursos de 80 a 90 horas lectivas, ya que, si la cantidad de estudiantes es mayor o el número de horas menor, se debería medir el efecto de reducir el número de actividades a una de input y otra de práctica ó output.

Además, en términos de metodología de enseñanza, también deberán ser analizados, en futuros estudios, los contenidos pragmáticos que sería conveniente incluir en cada nivel de competencia.

\section{CONCLUSIONES}

Mediante la propuesta didáctica planteada, se busca incrementar tanto la conciencia pragmática de los aprendices en la lengua meta, como el uso de categorías de cortesía adecuadas a situaciones concretas. Por supuesto, su aplicación debe adecuarse el nivel de proficiencia de los $\mathrm{HnN}$ con los cuales se esté trabajando en el aula.

Los contenidos pragmáticos por desarrollar deben incluir información sobre los dos aspectos fundamentales de esta competencia: las estructuras lingüísticas y el componente socio-cultural en la lengua meta.

Acorde con lo planteado en Kasper (1997), el componente pragmático de la cortesía exige la elaboración de instrumentos pedagógicos específicos mediante los cuales el estudiante de segunda lengua se acerque eficazmente a la lengua meta en uso. El papel de la instrucción es brindar las oportunidades para desarrollarlo. 
Las implicaciones pedagógicas al incluir este componente son muy variadas, tanto para la enseñanza en el aula de L2 como para la elaboración de materiales, de planes de estudio o de libros de enseñanza del español como segunda lengua. Lo anterior, sobre todo si se piensa en un método que integre las distintas competencias desde los primeros estadios de aprendizaje, y no se quiera seguir incurriendo en el error de solo prestar atención a aspectos formales de la lengua o de pensarlos como principal vía para desarrollar competencia en una segunda lengua, dejando de lado el contenido sociocultural.

Afortunadamente, en los últimos años ha aumentado el número y la calidad de las investigaciones en aspectos pragmáticos del español, los actos de habla, la cortesía y los contextos sociales y culturales de uso de la lengua, aportes que pueden servir de base para la creación de instrumentos pedagógicos apropiados en el área del desarrollo de la competencia pragmática de los estudiantes de español como segunda lengua o lengua extranjera.

\section{NOTAS}

${ }^{1}$ La segunda lengua es la que se adquiere en un contexto en el que funciona como lengua nativa, por el contrario, si la lengua se aprende en un contexto ajeno a la lengua meta (la lengua por adquirir) se habla de adquisición de una lengua extranjera.

${ }^{2}$ Al llevar a cabo cualquier acto de habla (acción), Austin (1962) propone que se activan tres dimensiones:

a) un acto locutivo: el acto físico de emitir el enunciado.

b) un acto ilocutivo o intención: la realización de una función comunicativa como invitar, prometer, afirmar, etc.

c) un acto perlocutivo o efecto: la reacción que provoca la emisión en el interlocutor. Así, al emitir cualquier enunciado como "¿podés venir a casa esta noche?”, se dice algo (acto locutivo), se invita (acto ilocutivo) y se provoca un efecto: el interesar al interlocutor a aceptar o rechazar una invitación (acto perlocutivo).

${ }^{3}$ El enfoque comunicativo define la competencia comunicativa no solo como la habilidad lingüística de las aprendientes, sino como la de comunicarse adecuadamente en la lengua meta en contextos comunicativos específicos.

${ }^{4} \mathrm{La}$ interlengua es el sistema lingüístico de transición que resulta de la combinación, por parte del hablante, de su lengua materna con la segunda lengua que aprende. 
${ }^{5}$ Quesada Pacheco (1992), conforme con la distribución de ciertos rasgos fonéticos, morfológicos y léxicos particulares, plantea una división dialectal del español de Costa Rica en cinco zonas, una de ellas es la variedad vallecentraleña: "1. Valle Central: comprende las provincias de San José (menos el cantón de Pérez Zeledón), Cartago, además de las partes altas de las provincias de Heredia y Alajuela." (p.86)

${ }^{6}$ Con el fin de organizar los recursos discursivos en la investigación citada, se empleó el análisis de las categorías para clasificar expresiones de cortesía en corpus de lengua oral presentado por Bravo (2005, p.37), en el que se distingue "acto" para lo que en forma más evidente se entiende como el objetivo comunicativo que es hecho explícito en una contribución ( por ejemplo, aceptar o rechazar la invitación) y "subacto" cuando se puede interpretar otro objetivo que se deriva del primero( agradecer, solicitar información, etc.).

${ }^{7}$ El Marco de Referencia Europeo para el Aprendizaje, la Enseñanza y la Evaluación de Lenguas (2001) propone un sistema común de niveles de competencia lingüística partiendo de la división inicial en tres niveles amplios, A, B y C, que pueden inscribirse, en términos generales, en la línea del sistema tradicional de los niveles principiante, intermedio y avanzado. Cada uno de estos tres niveles amplios se subdivide en dos, hasta configurar el sistema completo.

${ }^{8}$ Ver Marco de referencia europeo para el aprendizaje, la enseñanza y la evaluación de lenguas: Capítulo 5. Las Competencias del Usuario o Alumno, específicamente los apartados relacionados con la competencia sociolingüística y la competencia pragmática.

\section{REFERENCIAS}

Austin, J. (1962). How to do things with words. Oxford: Oxford University Press.

Bardovi-Harlig, K. (2001). Empirical evidence of the need for instruction in pragmatics. En Rose, K. y Kasper, G. (eds.). Pragmatic development in a second language. Michigan: Blackwell Publishing.

Bravo, D. (Ed.). (2003). Primer coloquio del Programa EDICE. Estocolmo: Universidad de Estocolmo.

Brown, P.; Levinson, S. (1987). Politeness, some universal in language use. Cambridge: Cambridge University Press.

Dumitrescu, D. (2005). Agradecer en interlengua: una comparación entre la competencia pragmática de los estudiantes nativos y no nativos del español en California. En Diana Bravo (Ed.), Estudios del discurso de cortesía en español. II Coloquio del programa EDICE. San José, Costa Rica: Universidad de Costa Rica.

Ellis, R. (1992). Learning to communicate in the classroom: A study of two learners'requests. Studies in Second Language Acquisition, 14, 1-23.

Escandell, V. (1995a). Cortesía, fórmulas tradicionales y estrategias indirectas. Revista Española de lingüística, 25 (1), 31-66.

Vidal, V. (1996b). Introducción a la Pragmática. Barcelona: Editorial Ariel. 
Ferrer, M. C.; Sánchez Lanza C. (2000). Actos de habla comisivos: la invitación. Revista Argentina de Lingüística, 16, 35-59.

Grice, H.P. (1975). Logic and conversation. En P.Cole y J.L.Morgan (Eds.), Syntax and Semantic: Speech Acts. Nueva York: Academic Press.

Haverkate, Henk. (1994). La cortesía verbal. Madrid: Editorial Gredos.

Kasper, G.; Dahl. (1991a). Research methods in interlenguage pragmatics. Studies in second language acquisition, 13, 215-247.

, G.; Kenneth R. (2001b). Pragmatics in language teaching. En Long, M. and Jack Richards (Eds.), The Cambridge Aplied Linguistics Series. Cambridge University press.

, G. (2001c). Four perspectives on L2 pragmatic development. Applied

Linguistics, 22 (4), 502-525.

, Gabriele; Kenneth R. (2002d). Pragmatic development in a second language. Language learning. A Jurnal of Research in Languaje Studies, 52 (1).

Leech, G.N. (1983). Principles of pragmatics. London: Longman.

Levinson, S.C. (1983). Pragmatics. Cambridge: Cambridge University Press.

Llobera et al. (1995). Competencia comunicativa. Documento básico en la enseñanza de lenguas extranjeras. Madrid: Edelsa.

Murillo, J. (2004a). La pragmática y la enseñanza del español como segunda lengua. Revista de Educación, 28 (2), 253-266. , Jorge. (2003b). La cortesía verbal en el español de Costa Rica. Revista Káñina, 26 (2). Universidad de Costa Rica.

Piatti, G. (2003). La cortesía: un contenido funcional para los programas de español como lengua extranjera. En Bravo (Ed.), I Coloquio del programa EDICE (pp. 355-397). Estocolmo, Suecia.

Quesada, M. A. (1992). Pequeño atlas lingüístico de Costa Rica. Revista de Filología, Lingüística y Literaturade la Universidad de Costa Rica. 18(2).

Sánchez, A. (1997). Los métodos de la enseñanza de idiomas. Madrid: Sociedad General Española de Librerías S.A.

Searle, J.R. (1975). Indirect speech acts. In Cole, P. y Morgan, J.L. (Eds.), Syntax and Semantics 3: Speech Acts. Nueva York: Academic Press.

Tateyama, Y. ; Kasper, G. ; Mui, L. ; Tay, H. and Thananart, O. (1997a). "Explicit and implicit 
teaching of pragmatics routines". In L. Bouton (Ed.), Pragmatics and language learning, (8). Urbana, IL: University of Illinois at Urbana-Champaign.

, Y. (2001b). "Explicit and implicit teaching of pragmatics routines: Japanese sumimasen". In G. Kasper y Kenneth Rose (eds.), Unpublished doctoral dissertation (pp.200-223). University of Hawwai'i at Manoa.

Thomas, J. (1983). Cross-cultural pragmatic failure. Applied Linguistics, 4, 91-112.

Urbina, M.S. (2004a). Análisis pragmático de dos expresiones de cortesía del español de Costa Rica y su adquisición por parte de estudiantes de español como Segunda Lengua. Revista de Filología y Lingüística de la Universidad de Costa Rica, 30(2), 237-245

, M.S. (2007b). Aceptar y rechazar una invitación. Estudio comparativo de la competencia pragmática de estudiantes de español como segunda lengua. Tesis de Maestría. Universidad de Costa Rica.

\section{Fuentes de Internet}

Bani, S.; Nevado A. (2004). Aproximaciones a la cortesía verbal en manuales de E/LE. Artifara N. 4 (enero - junio 2004), sección Monográfica. Recuperado el 16 de marzo de 2008, de http://www.artifara.com/rivista4/testi/cortesía.asp.

Bardovi-Harlig, K.; Mahan-Taylor, R. (2003). Teaching pragmatics. Washington DC: U.S. Department of State Office of English Language Program. Recuperado el 19 de abril de 2008, de http://exchanges.state.gov/education/engteaching/pragmatics.htm

Escandell Vidal, V. (En prensa). Enseñar español como segunda lengua L2 / lengua extranjera. Vademécum para la formación de profesores, Madrid, SGEL. Recuperado el 29 de octubre de 2007, de http://www.uned.es/dpto-leng-esp-y-ling-gral/escandell/papers/AportPrag.PDF

Instituto Cervantes. (2001). Marco de referencia europeo para el aprendizaje, la enseñanza y la evaluación de lenguas. (Versión traducida al español). Recuperado el 15 de marzo de 2008, de http://cvc.cervantes.es/obref/marco (versión electrónica del documento).

Kasper, G. (1997). Can pragmatic competence be taught? Honolulu: University of Hawaii. Second Language Teaching and Curriculum Centre. Recuperado el 23 de mayo de 2008, de http://www.nflrc.hawaii.edu/NetWorks/NW06/

Miranda Ubilla, H. (2000). La cortesía verbal en textos para la enseñanza del español e inglés como lenguas extranjeras. Tesis doctoral. Recuperado el 23 de mayo de 2008, de http://www.cervantesvirtual.com 
